\title{
Design of a Millimeter Wave Microstrip Patch Antenna using 52GHz for Mobile Communication
}

\author{
Wisdom Anuhu ${ }^{1}$, A. S. Husainni ${ }^{1,2,3}$, I.T .E. Elfergani ${ }^{2}$, B. A. Mohammed ${ }^{3}$, A. Salisu ${ }^{4}$, Majidi \\ Ibrahim Alashhb ${ }^{5}$, R.A. Abd-Alhameed ${ }^{3}$, J. Rodriguez $^{2,6}$ \\ ${ }^{1}$ School of Engineering, American University of Nigeria, Yola \\ \{wisdom.anuhu, ash.hussaini\}@aun.edu.ng \\ ${ }^{2}$ Instituto de Telecomunicações - Aveiro, Portugal \\ \{i.t.e.elfergani, ash, jonathan\}@av.it.pt \\ ${ }^{3}$ University of Bradford, Bradford, BD7 1DP, UK, \\ \{r.a.a.abd, a.s.hussaini7\} @bradford.ac.uk \\ ${ }^{4}$ Department of Electrical Engineering, Modibbo Adama University of Technology, Yola \\ abubakarsalisu06@mautech.edu.ng \\ ${ }^{5}$ Higher Institute of Engineering Technology Zliten, Libya. \\ alashhbmajdi@gmail.com \\ ${ }^{6}$ University of South Wales, Pontypridd CF37 1DL, UK \\ jonathan.rodriguez@southwales.ac.uk
}

\begin{abstract}
The 5G technology is one designed to solve the problem of bandwidth shortage. The design process of the state of the art $5 \mathrm{G}$ antenna will be in prospect to the $5 \mathrm{G}$ specifications. The proposed antenna is an array of microstrip patch antenna with a dielectric substrate $\left(\mathrm{E}_{\mathrm{r}}=0.4\right)$. The microstrip patch antenna is designed to operate on a millimeter wave with a frequency of $52 \mathrm{GHz}$. This paper is centered on the design of the single radiating element (patch) rather than the array microstrip patch antenna. However, further analysis for the design of the microstrip patch antenna to support Massive Input, Massive Output (MIMO) and beamforming will be discussed.
\end{abstract}

Keywords: MIMO, mm-wave, 3GPP, 5G.

\section{Introduction}

The telecommunication industry is undergoing a rapid change constantly. These changes are as a result of the need to create or improve the existing communication network and also to satisfy the requirements of emerging technologies. The $3^{\text {rd }}$ Generation Partnership Project (3GPP) has been at the core of the development of the $5^{\text {th }}$ Generation of mobile communication. Every year they release specifications, protocols and architecture called release documents to 
enable researchers and engineers in the industry think in line with those recommendations and develop models or solutions that can be used. In all the release documents on 5G networks, key characteristics such as low latency, ubiquitous coverage, high capacity and ultra-experience has been over emphasized. In that regard, the microstrip patch antenna discussed in this paper is one that supports the aforementioned characteristics. The choice of this particular antenna is based on the fact that it has low profile, low cost and they are suitable for linear and planar surfaces [2]. Several researches in antenna design suggest that the antenna should have a high gain for penetrating capabilities, compact dimensions to reduce the effect of coupling, narrow bandwidth, efficiency and multiband [6]. In order to design an antenna that will support emerging technologies and provide high capacity \& ultra-experience, the antenna will make use of the mm-wave with a frequency of $52 \mathrm{GHz}$. The choice of this frequency is purely based on the suitable results obtained at this band in terms of bandwidth and gain. However, other bands which support mm-wave are $28 \mathrm{GHz}, 37 \mathrm{GHz}, 39 \mathrm{GHz}$ and $64-71 \mathrm{GHz}$ [3].

Massive Input, Massive Output (MIMO) is an important technique employed into the design of this antenna to improve latency as well as ubiquitous coverage. However, mutual coupling is a factor that mostly affect this kind of antenna design at high frequency. But in order to minimize this effect, a common practice done is by introducing a defect in the ground section of the microstrip antenna [5].

One of the major problem in designing the $5 \mathrm{G}$ antenna is the ability to obtain a high bandwidth that IoT devices can be able to use. Paritosh and Ashwin in their paper "performance enhancement of millimeter wave antenna with integrated inter-digital capacitor structure" used a frequency of $30 \mathrm{GHz}$ to design their antenna to support $5 \mathrm{G}$ and it can provide a bandwidth of $1 \mathrm{GHz}$ [11]. Similarly, researchers; Qian, Ning, LingLi, Safiddin and JingPing in their paper "5G MIMO conformal Microstrip Antenna Design" used 35GHz frequency to design their antenna also having a bandwidth of $1 \mathrm{GHz}$ [10]. In a research conducted by Muhammad and Majeed in the paper "Millimeter-Wave Pattern Reconfigurable Antenna" used a frequency of $79 \mathrm{GHz}$ and still obtained a bandwidth of $1 \mathrm{GHz}$. This same result was obtained by Igor, Shual and Pedersen in works titled "Dual-Polarized Dual-band Mobile 5G Antenna Array" which operated on a frequency of $33.5 \mathrm{GHZ}$ [12].

At end of this research I discovered that a higher bandwidth can be obtained when a frequency of $52 \mathrm{GHz}$ was used to design the antenna (preferably, the microstrip patch). The bandwidth obtained is about $3 \mathrm{GHz}$ which is a massive boost to what have been previously obtained.

\section{Design}

As identified by many researchers as well as antenna design engineers, the three key parameters to pay close attention to in designing a microstrip patch antenna are: dielectric constant, loss tangent and the height of the substrate. More to it, the desired bandwidth and radiation efficiency can be obtained by careful computation of the width (W) as well as the Length (L) of the patch [4], [7], [8],[10]. The selected height of the substrate is $1 \mathrm{~mm}$, dielectric constant $\mathrm{E}_{\mathrm{r}}=0.4$ and operating frequency is $52 \mathrm{GHz}$.

The length and width can be obtained using the formulas (1) and (2) respectively:

$$
\begin{aligned}
\mathrm{L} & =\frac{C o}{2 F r \sqrt{E r}} \\
\mathrm{~W} & =\frac{C o}{2 F r \sqrt{E r} / 2}
\end{aligned}
$$


Where $\mathrm{C}_{\mathrm{o}}=$ speed of light

$\mathrm{F}_{\mathrm{r}}=$ frequency

$\mathrm{E}_{\mathrm{r}}=$ dielectric constant

Applying the formulas above, the length obtained is about $3.2 \mathrm{~mm}$ and width is $5.0 \mathrm{~mm}$. In

Figure 1 below, the design of the microstrip patch antenna is shown.

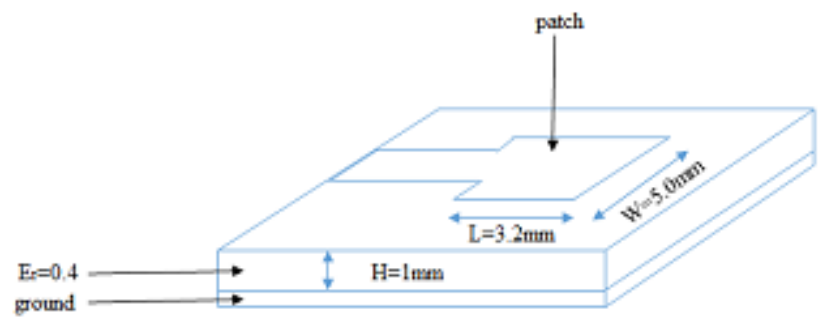

Fig. 1. The microstrip patch antenna design

\section{Result (Return loss, Bandwidth and Radiation)}

HFSS was used to obtain the simulation results. Figure 2 shows the reflection coefficient $\mathrm{S}_{11}$ or the return loss of $-38 \mathrm{~dB}$ at $52 \mathrm{GHz}$. Figure 3 shows the bandwidth obtanined with frequency sweep between $48 \mathrm{GHz}$ and $58 \mathrm{GHz}$ which resulted in a bandwith of about $3 \mathrm{GHz}$. While figure 4 shows the radiation pattern with peak gain of $5 \mathrm{~dB}$.

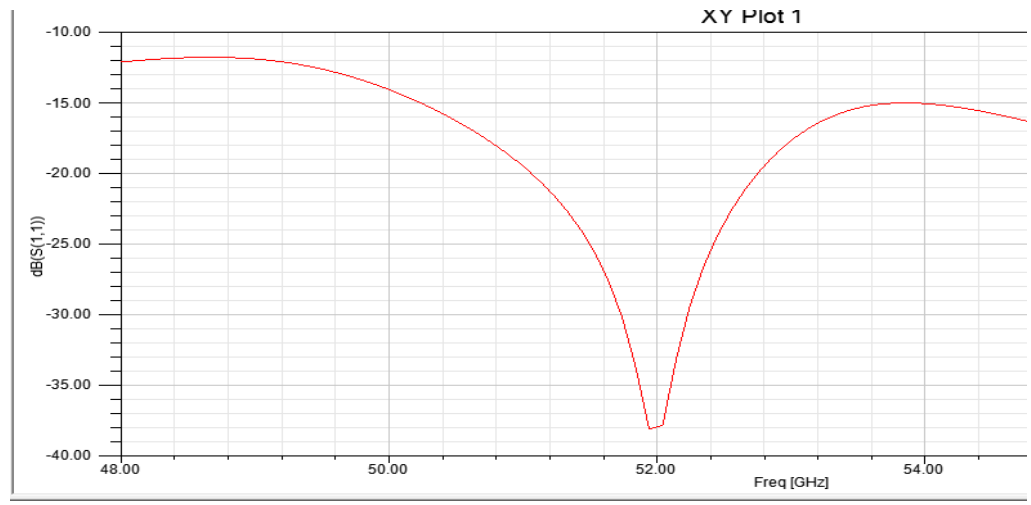

Fig. 2. The S11 plot which show the return loss 


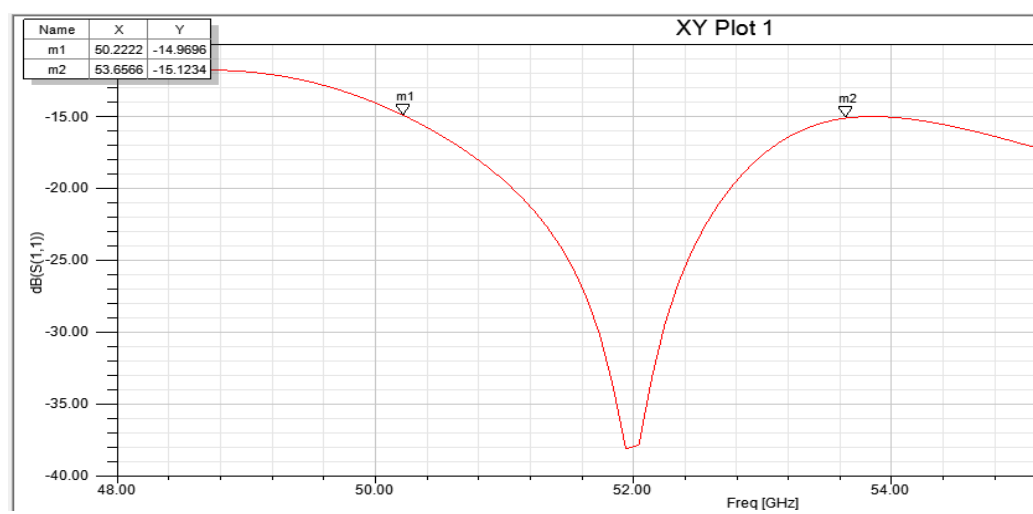

Fig. 3. Show the bandwith obtained at markers M1 and M2

$$
\begin{aligned}
& \text { dB(GainTotal) }
\end{aligned}
$$

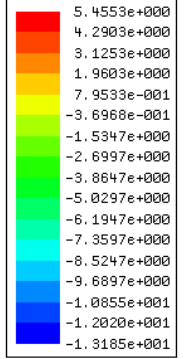

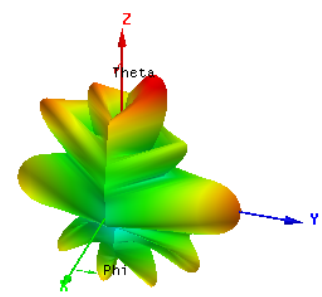

Fig. 4. Radiation 3D polar 


\section{MIMO}

The current frequencies at which various electronic devices operate on is less than or equal to $3 \mathrm{GHz}$. At this frequency band, it will be extremely difficult to deploy IoT devices which require about $1 \mathrm{GHz}$ of bandwidth. Additionally, this frequency band will not accommodate the anticipated number of devices that will operate on $5 \mathrm{G}$. Therefore, researches and experts proposed the mm-Wave which will brought another dimension with respect to speed, bandwidth and network capacity. As identified above, the mm-wave operates on a very high frequency (between $28 \mathrm{GHz}$ and $73 \mathrm{GHz}$ ) and at this frequency, signal fading as often experienced [5]. In order to solve this problem, the Multiple Input Multiple Output technique which was employed in the LTE will be used in the designing 5G antennas. The MIMO technique involves using arrays of antennas which will operate concurrently at the same frequency to transmit and receive data. Typically, the transmission channel is multiplex to carry different types of data. The type of multiplexing proposed for the $5 \mathrm{G}$ technology is the spatial multiplexing.

The spatial multiplexing has been widely used in wireless communication. One fundamental characteristic of this type of multiplexing is the ability to increase the data rate on a channel capacity. Shannon expressed that data cannot be transmitted or data will be compromised with errors if it is transmitted above the theoretical boundary expressed in the equation (1) below. What spatial multiplexing does is to increase the data rate of a multiplex channel without increasing power, which cannot be achieved when using a single channel. It is established that in any MIMO multiplexing the number of transmitting antennas must be equal to the number of receiving antennas since the transmission channel is divided into streams of different data types. The relationship between the transmitted signal and the received signal in spatial multiplexing is expressed as a system of liner equations. Below in Figure 5 is a diagram that shows how data is transmitted and received in spatial multiplexing as well as the system of linear equations

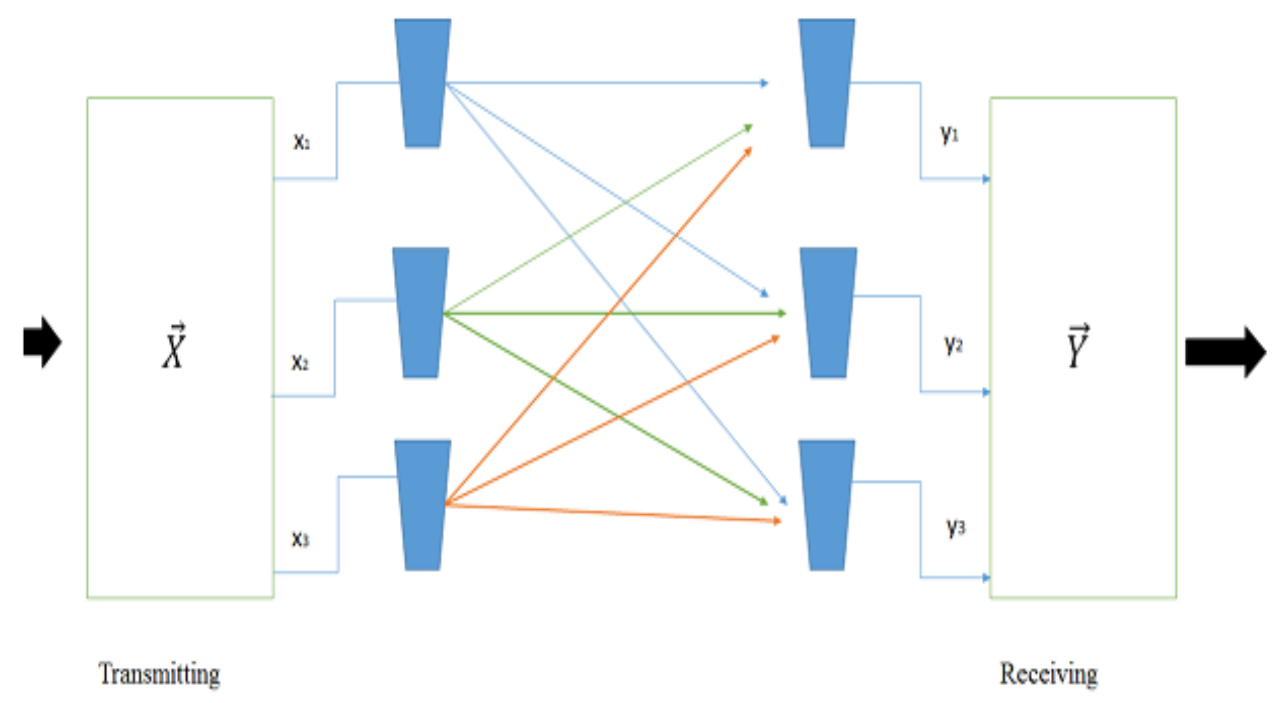

Figure 5: Spiral multiplexing diagram 
$\vec{Y}=\left[\begin{array}{ccc}h 11 & \cdots & h 31 \\ \vdots & \ddots & \vdots \\ h 31 & \cdots & h 33\end{array}\right] \vec{X}$

Where $\vec{Y}=$ received signal

$\vec{X}=$ Transmitted signal

$\mathrm{h}=$ data

\section{Beamforming}

The most effective $5 \mathrm{G}$ antenna will be one that utilizes the beamforming technique. Beamforming is most times used to describe an antenna as "smart". The two types of smart antennas are switch beam and adaptive antenna. The switch beam operates by directing the desired signal to a device that moves from one cell to the other [9]. The adaptive smart antenna which is often regarded as the best type of antenna, directs its main lobe to a device as it moves from one cell to the other by discarding the signal not of interest (SNOI) and hence reducing inteference [1]. The microstrip patch antenna discussed in this paper can also be made adaptive by introducing a digital signal processing mechanism on it.

\section{Conclusion}

The microstrip patched antenna discussed in this paper was one designed to meet the requirements of the $5 \mathrm{G}$ and other emerging technologies that comes with it. Several techniques introduced such as the MIMO and the use of mm-wave were engineered to meet the 3GPP 5G recommendations. Introducing beamforming is also a crucial aspect of antenna design which is looked upon to further enhance the strength of the state of the art $5 \mathrm{G}$ antenna. Although the microstrip patch antenna discussed in this paper was not designed with beamforming capabilities, it can still be implemented. The best $5 \mathrm{G}$ antenna to be used would be one that is cheap, has low profile and implemetns most of the requirements proposed by the 3GPP. 


\section{References}

[1] Balanis, Constantine A. Antenna Theory Analysis and Design. New Jersey : John Wiley \& Sons, 2005.

[2] Chauhan, Brajlata and Vijay, S.C., Gupta Sandip. "Millimeter-Wave Mobile Communications." International Journal of Computer Applications (2014): 1-5.

[3] Gampala, Gopinath and C. J. Reddy. "Design of Millimeter Wave Antenna Arrays for 5G." IEEE (2016): 1-2.

[4] Joshi, Sanket, et al. "Effect of Mutual Coupling on Microstrip Antenna." Procedia Computer Science (2015): 1-5.

[5] Maharjan, Janam, Kim Sun-Woong and Choi Dong-You. "Franklin Array MIMO Antenna for 5G Applications." (2017): 1-4.

[6] PESHWE, Paritosh and Ashwin KOTHARI. "Performance Enhancement of Millimeter Wave Antenna." Radioengineering (2018): June. 1-7.

[7] Pimpalgaonkar, Pratima R, et al. "A Review on Dielectric Resonator Antenna." 1ST INTERNATIONAL CONFERENCE ON AUTOMATION IN INDUSTRIES (ICAI) (2016): 13.

[8] RASHID, MD MAMUNUR and HOSSAIN SADDAM. "Antenna Solution for Millimeter Wave Mobile." International Journal of Scientific Research Engineering \& Technology (IJSRET) (2014): 1-4.

[9] Saleem, Muhammad Kamran, A. S. Alkanhal Majeed and Fattah Sheta Abdel. "SWITCH BEAM DIELECTRIC RESONATOR ANTENNA ARRAY WITH FOUR RECONFIGURABLE RADIATION PATTERNS." MICROWAVE AND OPTICAL TECHNOLOGY LETTERS / (2016): 1-6.

[10] Wang, Qian, et al. "5G MIMO Conformal Microstrip Antenna Design." Wireless Communications and Mobile Computing (2017): 1-11.

[11] Peshwe, Paritosh and Kothari Ashwin. "Performance Enhancement of Millimeter Wave Antenna." ELECTROMAGNETICS (2018): 1-8.

[12] Syrytsin, Igor, Shuai Zhang and G.F. Pedersen. "Dual-polarized Dual-band Mobile 5G Antenna Array." Proceedings of the 15th International Joint Conference on e-Business and Telecommunications (ICETE 2018). International Joint Conference on E-business and Telecommunication Networks, 2018. 1-9. 